\title{
Capsule Commentary on Newman et al., Yelp for Prescribers: A Quasi-Experimental Study of Providing Antibiotic Cost Data and Prescription of High-Cost Antibiotics in an Academic and Tertiary Care Hospital
}

\author{
Hyung (Harry) J Cho, MD \\ Division of Hospital Medicine, Department of Medicine, Mount Sinai Hospital, New York, NY, USA.
}

J Gen Intern Med 30(8): 1188

DOI: $10.1007 / \mathrm{s} 11606-015-3308-4$

(c) Society of General Internal Medicine 2015

$\mathrm{T}$ he trial by Newman et al. ${ }^{1}$ integrated into the EHR antibiotic cost data with antibiotic susceptibility results related to cultures from bacteremia or urinary tract infections. The study showed a significant decrease in overall unit antibiotic cost per patient.

As shown in a recent analysis by Goetz et al., ${ }^{2}$ evidence exists for integrating cost data into the EHR in reducing overall costs. This study by Newman et al. has shown a benefit in reducing costs in antibiotic treatment utilizing two interesting innovations: a cost scale and a quality scale. Much like Yelp, which shows information about a business using a scale for cost and quality (determined by $\$$ signs and stars, respectively) this study showed a benefit using $\$$ signs and susceptibility testing. This has great implications for health care, as providers function much like users on Yelp; they are busy and need quick comparisons in a language that is easy to understand. Further studies are needed to determine whether these scales can be translated to other areas of EHR, as the comparisons can get challenging. For example, utilizing it in a small subset of beta-blockers may be easier than comparing all radiologic tests, as the number of tests and the variation in cost and indications can be high. Health care has a long history of borrowing designs from other fields to effect quality or value improvement (i.e. lean manufacturing from Toyota, or Ishikawa diagrams from Kawasaki). This may be another transferrable standard for improvement.

More research is needed in utilizing cost data within EHRs. This study's intervention is simple, cost-effective, and seamlessly integrated into the EHR. It is non-obstructive to daily workflow and does not create a hard stop or pop-up, which can lead to alarm fatigue. It is easily transferable to other institutions and EHRs. However, the study design could benefit from a control and was sensitive to unmeasured bias. The mean length of stay is very high (16.3 and 15.8), is not reflective of most hospitalizations, and begs the question, is this beneficial for shorter length of stays? Additionally, can this help with other infections?

Corresponding Author: Hyung (Harry) $J$ Cho, MD; Division of Hospital Medicine, Department of Medicine, Mount Sinai Hospital, New York, NY 10021, USA (e-mail: hyung.cho@mountsinai.org).

\section{REFERENCES}

1. Newman, Kira L, Varkey Jay, Rykowski Justin, Mohan Arun V. Yelp for Prescribers: A Quasi-Experimental Study of Providing Antibiotic Cost Data and Prescription of High-Cost Antibiotics in an Academic and Tertiary Care Hospital. J Gen Int Med. 2015;1-7. doi:10.1007/s11606-0153253-2

2. Goetz, Celine, Rotman Stephen R, Hartoularos George, Bishop Tara F. The effect of charge display on cost of care and physician practice behaviors: a systematic review. J Gen Int Med. 2015;1-8.

Published online April 11, 2015 